\title{
Acupuncture therapy for radiotherapy-induced adverse effects: a protocol for systematic review and Bayesian network meta-analysis
}

\author{
Tong Wu ${ }^{1 \# \wedge}$, Chengwei Fu ${ }^{2 \#}$, Yiran Deng ${ }^{1 \wedge}$, Wanping Huang ${ }^{1 \wedge}$, Xiaoxiao $\mathrm{Li}^{3}$, Yang Jiao ${ }^{4,5}$ \\ ${ }^{1}$ College of Acupuncture and Orthopedics, Hubei University of Chinese Medicine, Wuhan, China; ${ }^{2}$ The Second Clinical Medical School, \\ Guangzhou University of Chinese Medicine, Guangzhou, China; ${ }^{3}$ Cardiac Function Room, Wuhan Wuchang Hospital, Wuhan, China; ${ }^{4}$ Hubei \\ Provincial Hospital of Traditional Chinese Medicine, Wuhan, China; ${ }^{5}$ Hubei Province Academy of Traditional Chinese Medicine, Wuhan, China \\ \#These authors contributed equally to this work. \\ Correspondence to: Prof. Yang Jiao, BD. Hubei Provincial Hospital of Traditional Chinese Medicine, Wuhan, 430061, China; Hubei Province Academy \\ of Traditional Chinese Medicine, Wuhan 430074, China. Email: jiaoyang@hbhtcm.com.
}

Background: Radiotherapy is the cornerstone in cancer treatment, and its adverse effects have been
recognized widely nowadays. In response, effective and nontoxic therapies are in demand for patients
affected by radiotherapy-induced adverse effects (RIAE). As a multitude of clinical studies have suggested
that acupuncture therapies seem to be potential in treating RIAE, this study aims to make a systematic review
and Bayesian network meta-analysis (NMA) to evaluate effectiveness and safety of different acupuncture
treatments.

Methods: A full-scale search will be performed in the following databases from inception to June, 2020: PubMed/Medline, Cochrane library, Web of Science, Ebsco, Embase, China National Knowledge Infrastructure (CNKI), Wanfang Database, VIP Database and China Biology Medicine disc (CBM). Randomized controlled trials meeting the eligible criteria based on PICOS elements will be included. The primary outcome is the response rate of RIAE or the incidence of RIAE. The secondary outcome is the incidence of adverse events directly related to acupuncture. Cochrane risk-of-bias tool (ROB 2.0) will be employed to evaluate the quality of chosen literatures. Stata, Addis and OpenBUGS will be performed to manage data.

Discussion: The results can provide a relatively objective evidence to assess effectiveness and safety of acupuncture therapies for each RIAE, which may be rewarding as a guiding proposal for researchers concerning RIAE.

Trial registration: This study has been registered at INPLASY (https://inplasy.com/) with a registration ID INPLASY202070054.

Keywords: Acupuncture therapy; radiotherapy-induced adverse effects (RIAE); network meta-analysis (NMA)

Submitted Aug 31, 2020. Accepted for publication Dec 17, 2020.

doi: 10.21037/apm-20-1747

View this article at: http://dx.doi.org/10.21037/apm-20-1747

\footnotetext{
^ ORCID: Tong Wu, 0000-0003-3103-7909; Chengwei Fu, 0000-0002-2185-0560; Yiran Deng, 0000-0003-4342-3778; Wanping Huang, 0000-0002-5598-3521.
} 


\section{Introduction}

Radiation therapy is invariably regarded to be pivotal in treatment of cancers, which has been used in more than $50 \%$ of patients with cancer, for both curative and palliative purposes $(1,2)$. It undoubtedly comes with radiotherapy-induced adverse effects (RIAE) for cancer patients (3). Adverse effects decrease patients' life quality and even cause a treatment withdrawal (4). Reducing the radiation dose in normal tissue can control adverse effects, but it brings with low efficiency in the meantime (5-7). Current medications for RIAE either mark adverse effects of their own, or lack of proven specificity (8-11). As an example of pharmacological interventions for radiationinduced salivary gland dysfunction, insufficient evidence determined the effects of interventions like pilocarpine, bethanechol and palifermin (12). Given a long-lasting adverse symptom, coupled with long duration of therapy and expense suggest more promising options, both psychologically and economically (13).

As complementary and alternative therapies, acupuncturerelated interventions have been proved positive in treating RIAE these years (14-16). Under the guidance of traditional Chinese medicine (TCM), radiation is regarded as an external toxicity and correspondingly acupuncture therapies can strengthen the body resistance to eliminate pathogenic factors $(17,18)$. Acupuncture is recommended for patients with xerostomia after radiotherapy $(19,20)$. One largesample meta-analysis described moxibustion can reduce gastrointestinal toxicities of radiotherapy (21). Despite several researches held discrepant attitudes towards different acupuncture therapies (22-24), an updated review could be feasible as researchers have gradually poured attention to this topic. Therefore, we hold the ambition for assessing the efficacy and safety of various acupuncture therapies for different RIAE by performing a Bayesian network metaanalysis (NMA).

We present the following article in accordance with the PRISMA reporting checklist (available at http://dx.doi. org/10.21037/apm-20-1747).

\section{Methods}

The Preferred Reporting Items for Systematic review and Meta-Analysis Protocols (PRISMA-P) statement will be performed in this article $(25,26)$.

\section{Eligible criteria}

\section{Types of studies}

Randomized control trials (RCTs) in peer-reviewed journals will be included. Languages are limited to Chinese and English. Reviews, retrospective studies, case reports, protocols, animal studies, unpublished reports, studies unrelated to acupuncture and RIAE will be excluded. The most informative and complete one of duplicate studies will be included.

\section{Types of participants}

Patients received radiotherapy or individuals diagnosed as RIAE will be included, regardless of the type of cancer. Adverse events are embodied in systemic symptoms and local responses, as examples of myelosuppression and xerostomia.

\section{Types of interventions}

Acupuncture therapies are listed as interventions, including manual acupuncture, electroacupuncture, moxibustion, acupoint injection, auricular acupuncture, catgut embedding, transcutaneous electrical stimulation and so on. Acupuncture therapies with or without medication will also be counted in. Complete and precise acupoint prescriptions must be described in the literature while there is no limitation in acupoint selection. An example of possible network plot is displayed in Figure 1.

\section{Types of comparisons}

The comparisons contain placebo, usual care and medication. Placebo refers to sham acupuncture in most cases while usual care means routine clinical care. Besides, relevant common treatments are also used to treat different adverse events in control groups, which is a rational research design thus such circumstances should be accepted. Take the case of radiation enteritis, enema can be adopted as the comparison treatment. Complementary therapies unconnected with acupoints as comparisons like dietary therapy and aromatherapy will be excluded.

\section{Types of outcome measures}

\section{Primary outcome measures}

The primary outcome will be the response rate or the incidence of RIAE. Different RIAE will be discussed separately. Here are 2 examples following based on a pre- 


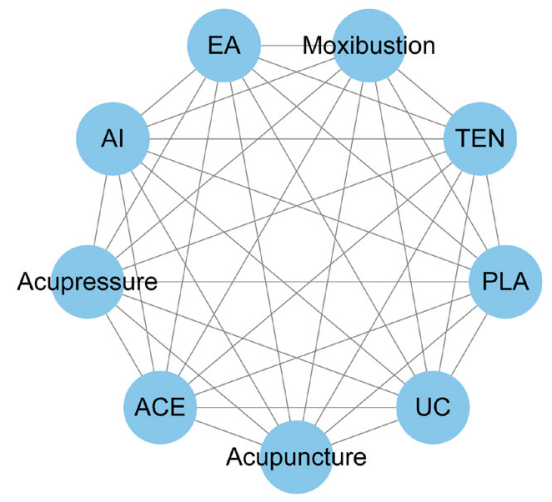

Figure 1 Network plot of possible direct comparisons. TEN, transcutaneous electrical stimulation; EA, electroacupuncture; AI, acupoint injection; ACE, acupoint catgut embedding; PLA, placebo; UC, usual care.

search: studies about vomiting and/or nausea caused by radiotherapy regard incidence of vomiting/nausea as the main outcome; studies about xerostomia caused by radiotherapy regard salivary flow rate as the main outcome.

\section{Secondary outcome measures}

The incidence of adverse events directly related to intervention as reported to evaluate safety measures will be regarded as the secondary outcome.

\section{Search strategy}

PubMed/Medline, Cochrane library, Web of Science, Ebsco, Embase, China National Knowledge Infrastructure (CNKI), Wanfang Database, VIP Database and China Biology Medicine disc (CBM) will be searched from inception to June 2020. The following search keywords and mesh terms will be used: radiotherapy ("radiotherapy" or "radiation therapy" or "cranial irradiation" or "Radiotherapy, Computer-Assisted" and so on); acupuncture ("acupuncture" or "electroacupuncture" or "acupuncture therapy" and so on). The search strategy of different databases will be modified properly. Appendix 1 shows a detailed search strategy for PubMed.

\section{Screening and selection of the RCTs}

For the first turn, all searched studies will be independently skimmed through the titles and abstracts by 2 reviewers, under the guidance of Appendix 2. Secondly, the fulltext reading of filtered studies will be accomplished to an elaborate selection. The third reviewer will make the final judgement if controversial. Moreover, when full text of literature can't be found after full-scale search, we will contact with the corresponding author. If we fail to get the full information, the data of this literature will be deleted. The selection process will be carried out in PRISMA flow chart as Figure 2.

\section{Data extraction and management}

A standard data extraction excel file will be created in the wake of targeted RCTs, with following information: (I) literature information: title, authors, the country of first author, year of publication, ethical approval and registration state; (II) patient information: sex, sample size, types of cancer, radiation dose, RIAE, types of intervention, types of comparison, intervention time point, endpoints, acupoints; (III) outcome information: the response rate of RIAE, the incidence of RIAE, degree of symptom improvement, observation time points of outcome. Necessary details will be added in the process of data extraction. Two reviewers will complete the task and the third review will act as a referee in the context of ambiguity and divergence. Furthermore, GetData Graph Digitizer will be applied to acquire digital information from figures.

\section{Risk of bias assessment}

Cochrane risk-of-bias tool (ROB 2.0) will be used to assess the quality (27). There are 5 sources of bias including bias arising from the randomization process, bias due to deviations from intended interventions, bias due to missing outcome data, bias in measurement of the outcome and bias in selection of the reported result. The final risk of bias can be figured out as 3 degrees: low risk, some concerns and high risk. Two reviewers will evaluate all filtered studies and the third reviewer will make the final decision if controversial.

\section{Data analyses}

\section{Pairwise meta-analysis}

The premise of Pairwise Meta-analysis is law of similarity, which means only when the included studies meet a certain degree of similarity, can the meta-analysis be carried out. We stipulate that no less than 3 studies of same interventions and outcome indicators can make sense in pairwise meta-analysis. With the tool of Stata 14.0, odds 


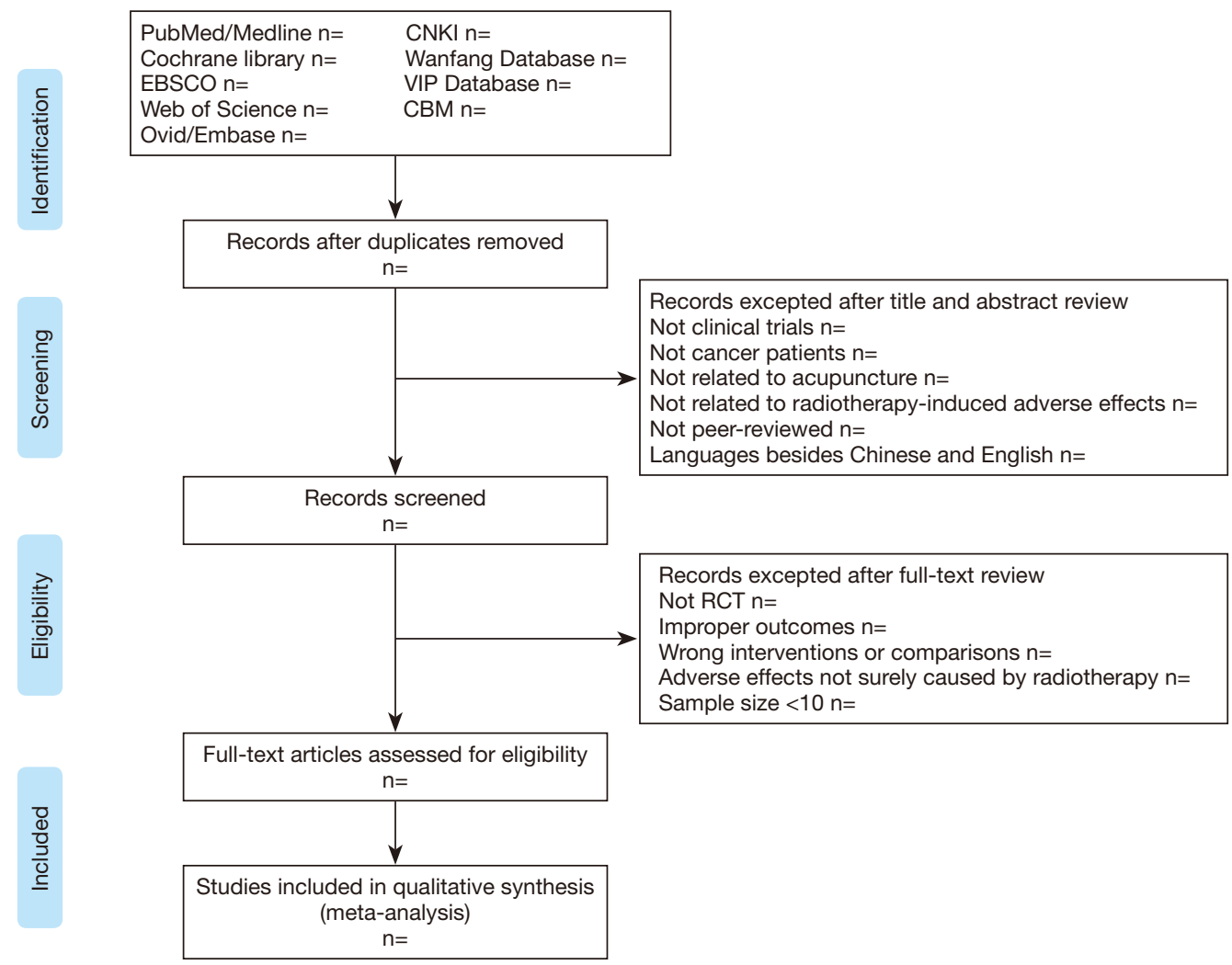

Figure 2 PRISMA flow diagram of the study selection process.

ratio (OR) and 95\% confidence interval (CI) are adopted. $\mathrm{I}^{2}$ test will be applied to assess heterogeneity and select model. A random effect model will be adopted if $\mathrm{I}^{2}>50 \%$ while a fixed effect model will be adopted if not. Before selecting model, sensitivity analysis will be performed properly to delete high-heterogeneity studies. By means of a Begg's testing, the reporting bias can be conducted if more than 10 pairwise comparison studies are included.

\section{Network meta-analysis}

NMA is the development of traditional meta-analysis. In this study, Addis1.16.8, OpenBUGS3.2.3 and Stata14.0 will be performed to present the results. OR and $95 \%$ CI will be adopted in the light of incidence, the outcome indicator, as dichotomous data. Addis1.16.8 evaluates the variance of chains by adopting convergence, with PSRF closed to 1 indicating a good convergence. If the $\mathrm{P}$ value $>0.05$, which means an excellent consistency, a consistency model will be used. As to inconsistency test for results, we will monitor the loop formed by studies with both direct evidence and indirect evidence, to figure out whether the inconsistency factor value approximate 0 . With a
95\% CI, it indicates a slim possibility of inconsistency if 0 is included, thus a consistency model should be also used. Besides, the surface under the cumulative ranking curve (SUCRA) values will carry out a possible range of interventions results by using OpenBUGS3.2.3, from 0 to $100 \%$. Considering that the outcomes of this study are incidences of RIAE, the closer the value is to $100 \%$, the worse the intervention effect will be.

\section{Subgroup analysis}

If related researches are sufficient, patients undergoing the same radiation dose can be regrouped in these studies. Therefore, it will bring with an analysis of acupuncturerelated therapies for adverse effects induced by different radiation dosage.

\section{Quality assessment}

The Grades of Recommendations Assessment Development and Evaluation (GRADE) guideline will be introduced to assess the quality of evidence. The guideline divides the quality of evidence to high, moderate, low, very low. 


\section{Ethics and informed consents}

Since this is a protocol for systematic literature research, ethical approval can be skipped. At the same time this protocol has been registered on INPLASY (https://inplasy. com/) with a registration ID INPLASY202070054.

\section{Discussion}

Radiotherapy is one of the three major treatments for cancer. According to a survey of WHO in 2017, it's estimated that about $45 \%$ of cancer could be cured, among which about $40 \%$ cured by radiotherapy (28). Obviously, cancer patients can get great benefit from radiotherapy if RIAE is under control. With an extensive application of acupuncture in clinical practice, researches concerning acupuncture therapy for RIAE appear in successions $(22,29)$. Modern technology has suggested that acupuncture can modulate neurological processes within the central nervous system (30), stimulate the autonomic nervous system and increase peripheral blood flow (16), which generally benefits patients undergoing radiation despite mechanism remains partly understood $(24,31)$. Since acupuncture theories and needling instruments gradually developed, a growing number of acupuncture therapies have emerged in the meantime, making clinical application a manifold yet tough decision as it remains inconclusive which treatment can reap the greatest benefits. Also, symptom management paired with type of therapies has remained to be studied. That's why we desire to conduct a network meta-analysis. Admittedly, there are some deficiencies existing in this review. The languages of literature are limited, which may lead to bias. Radiotherapy dose and acupoint prescription of acupuncture therapies will not be limited in eligible criteria, in this case, we will further present a subgroup analysis if permitting. The results of this protocol will be published in related journal, and a quick update will be made when supplements are required.

\section{Acknowledgments}

Funding: The authors have not declared a specific grant for this research from any funding agency in the public, commercial or not-for-profit sectors.

\section{Footnote}

Reporting Checklist: The authors have completed the
PRISMA checklist. Available at http://dx.doi.org/10.21037/ apm-20-1747

Conflicts of Interest: All authors have completed the ICMJE uniform disclosure form (available at http://dx.doi. org/10.21037/apm-20-1747). The authors have no conflicts of interest to declare.

Ethical Statement: Since no private and confidential patient data will be contained in the reporting, there are no ethical considerations associated with this protocol. This study has been registered at INPLASY (https://inplasy.com/) with a registration ID INPLASY202070054. The authors are accountable for all aspects of the work in ensuring that questions related to the accuracy or integrity of any part of the work are appropriately investigated and resolved.

Open Access Statement: This is an Open Access article distributed in accordance with the Creative Commons Attribution-NonCommercial-NoDerivs 4.0 International License (CC BY-NC-ND 4.0), which permits the noncommercial replication and distribution of the article with the strict proviso that no changes or edits are made and the original work is properly cited (including links to both the formal publication through the relevant DOI and the license). See: https://creativecommons.org/licenses/by-nc-nd/4.0/.

\section{References}

1. Citrin DE. Recent Developments in Radiotherapy. N Engl J Med 2017;377:1065-75.

2. Bentzen SM. Preventing or reducing late side effects of radiation therapy: radiobiology meets molecular pathology. Nat Rev Cancer 2006;6:702-13.

3. Panel's "Moonshot" Goals Released. Cancer Discov 2016;6:1202-3.

4. Shapiro CL. Highlights of Recent Findings on Qualityof-Life Management for Patients With Cancer and Their Survivors. JAMA Oncol 2016;2:1401-2.

5. Golden EB, Frances D, Pellicciotta I, et al. Radiation fosters dose-dependent and chemotherapyinduced immunogenic cell death. Oncoimmunology 2014;3:e28518.

6. Demaria S, Formenti SC. Radiation as an immunological adjuvant: current evidence on dose and fractionation. Front Oncol 2012;2:153.

7. Hoeller U. 5 - Radiotherapy-induced adverse events. W.B. Saunders 2011. 
8. Sanchiz F, Millá A, Artola N, et al. Prevention of radioinduced cystitis by orgotein: a randomized study. Anticancer Res 1996;16:2025-8.

9. Camidge R, Price A. Characterizing the phenomenon of radiation recall dermatitis. Radiother Oncol 2001;59:237-45.

10. Antonadou D, Coliarakis N, Synodinou M, et al. Randomized phase III trial of radiation treatment +/amifostine in patients with advanced-stage lung cancer. Int J Radiat Oncol Biol Phys 2001;51:915-22.

11. Sanguineti G, Franzone P, Marcenaro M, et al. Sucralfate versus mesalazine versus hydrocortisone in the prevention of acute radiation proctitis during conformal radiotherapy for prostate carcinoma. A randomized study. Strahlenther Onkol 2003;179:464-70.

12. Riley P, Glenny AM, Hua F, et al. Pharmacological interventions for preventing dry mouth and salivary gland dysfunction following radiotherapy. Cochrane Database Syst Rev 2017;7:CD12744.

13. Batumalai V, Wong K, Shafiq J, et al. Estimating the cost of radiotherapy for 5-year local control and overall survival benefit. Radiother Oncol 2019;136:154-60.

14. Braga FP, Lemos JC, Alves FA, et al. Acupuncture for the prevention of radiation-induced xerostomia in patients with head and neck cancer. Braz Oral Res 2011;25:180-5.

15. Moran MS, Ma S, Jagsi R, et al. A prospective, multicenter study of complementary/alternative medicine (CAM) utilization during definitive radiation for breast cancer. Int J Radiat Oncol Biol Phys 2013;85:40-6.

16. O'Sullivan EM, Higginson IJ. Clinical effectiveness and safety of acupuncture in the treatment of irradiationinduced xerostomia in patients with head and neck cancer: a systematic review. Acupunct Med 2010;28:191-9.

17. Cai HB, Luo RC. Prevention and therapy of radiationinduced pulmonary injury with traditional Chinese medicine. Di Yi Jun Yi Da Xue Xue Bao 2003;23:958-60.

18. Djaali W, Simadibrata CL, Nareswari I. Acupuncture Therapy in Post-Radiation Head-and-Neck Cancer with Dysgeusia. Med Acupunct 2020;32:157-62.

19. Johnstone PA, Niemtzow RC, Riffenburgh RH. Acupuncture for xerostomia: clinical update. Cancer 2002;94:1151-6.

20. Johnstone PA, Peng YP, May BC, et al. Acupuncture for pilocarpine-resistant xerostomia following radiotherapy for head and neck malignancies. Int J Radiat Oncol Biol Phys 2001;50:353-7.

21. Zhang HW, Lin ZX, Cheung F, et al. Moxibustion for alleviating side effects of chemotherapy or radiotherapy in people with cancer. Cochrane Database Syst Rev 2018;11:CD10559.

22. Garcia MK, McQuade J, Haddad R, et al. Systematic review of acupuncture in cancer care: a synthesis of the evidence. J Clin Oncol 2013;31:952-60.

23. Garcia MK, Chiang JS, Cohen L, et al. Acupuncture for radiation-induced xerostomia in patients with cancer: a pilot study. Head Neck 2009;31:1360-8.

24. Birch S, Lee MS, Alraek T, et al. Evidence, safety and recommendations for when to use acupuncture for treating cancer related symptoms: a narrative review. Integr Med Res 2019;8:160-6.

25. Hutton B, Salanti G, Caldwell DM, et al. The PRISMA extension statement for reporting of systematic reviews incorporating network meta-analyses of health care interventions: checklist and explanations. Ann Intern Med 2015;162:777-84.

26. Shamseer L, Moher D, Clarke M, et al. Preferred reporting items for systematic review and meta-analysis protocols (PRISMA-P) 2015: elaboration and explanation. BMJ 2015;350:g7647.

27. Sterne JAC, Savović J, Page MJ, et al. RoB 2: a revised tool for assessing risk of bias in randomised trials. BMJ 2019;366:14898.

28. Smith RA, Andrews KS, Brooks D, et al. Cancer screening in the United States, 2018: A review of current American Cancer Society guidelines and current issues in cancer screening. CA Cancer J Clin 2018;68:297-316.

29. Zhang Q, Sharan A, Espinosa SA, et al. The Path Toward Integration of Traditional and Complementary Medicine into Health Systems Globally: The World Health Organization Report on the Implementation of the 20142023 Strategy. J Altern Complement Med 2019;25:869-71.

30. Sagar SM. Acupuncture as an evidence-based option for symptom control in cancer patients. Curr Treat Options Oncol 2008;9:117-26.

31. Garcia MK, Meng Z, Rosenthal DI, et al. Effect of True and Sham Acupuncture on Radiation-Induced Xerostomia Among Patients With Head and Neck Cancer: A Randomized Clinical Trial. JAMA Netw Open 2019;2:e1916910.

Cite this article as: $\mathrm{Wu} \mathrm{T}, \mathrm{Fu} \mathrm{C}$, Deng $\mathrm{Y}$, Huang $\mathrm{W}$, Li $\mathrm{X}$, Jiao Y. Acupuncture therapy for radiotherapy-induced adverse effects: a protocol for systematic review and Bayesian network meta-analysis. Ann Palliat Med 2021;10(2):2254-2259. doi: 10.21037/apm-20-1747 


\section{Appendix 1}

The following shows a detailed search strategy for PubMed.

We choose the advanced search builder.

\begin{tabular}{|c|c|}
\hline \#1 & acupuncture[Title/Abstract] \\
\hline \#2 & acupoint*[Title/Abstract] \\
\hline \#3 & moxibustion[Title/Abstract] \\
\hline \#4 & "electric stimulation therap*"[Title/Abstract] \\
\hline \#5 & "auricular acupuncture"[Title/Abstract] \\
\hline \#6 & "transcutaneous electric nerve stimulation"[Title/Abstract] \\
\hline \#7 & "transcutaneous electric acupoint"[Title/Abstract] \\
\hline \#8 & electroacupuncture[Title/Abstract] \\
\hline \#9 & acupressure[Title/Abstract] \\
\hline$\# 10$ & "catgut embedding"[Title/Abstract] \\
\hline \#11 & "embedding therap*"[Title/Abstract] \\
\hline$\# 12$ & \#1 OR \#2 OR \#3 OR \#4 OR \#5 OR \#6 OR \#7 OR \#8 OR \#9 OR \#10 OR \#11 \\
\hline$\# 13$ & acupuncture[MeSH Terms] \\
\hline \#14 & "acupuncture therapy"[MeSH Terms] \\
\hline$\# 15$ & "acupuncture points"[MeSH Terms] \\
\hline$\# 16$ & moxibustion[MeSH Terms] \\
\hline$\# 17$ & electroacupuncture[MeSH Terms] \\
\hline$\# 18$ & "acupuncture, ear" [MeSH Terms] \\
\hline$\# 19$ & "transcutaneous electric nerve stimulation"[MeSH Terms] \\
\hline \#20 & "electric stimulation therapy"[MeSH Terms] \\
\hline \#21 & \#13 OR \#14 OR \#15 OR \#16 OR \#17 OR \#18 OR \#19 OR \#20 \\
\hline \#22 & $\# 12$ OR \#21 \\
\hline \#23 & "radiation therap*"[Title/Abstract] \\
\hline$\# 24$ & radiotherap*[Title/Abstract] \\
\hline \#25 & irradiation[Title/Abstract] \\
\hline \#26 & mammosite[Title/Abstract] \\
\hline \#27 & radiation treatment[Title/Abstract] \\
\hline \#28 & brachytherap*[Title/Abstract] \\
\hline$\# 29$ & chemoradiotherap*[Title/Abstract $]$ \\
\hline$\# 30$ & "x-ray therap*"[Title/Abstract] \\
\hline$\# 31$ & radiochemotherap*[Title/Abstract] \\
\hline$\# 32$ & radiodermatitis[Title/Abstract] \\
\hline \#33 & "radiation-induced oral mucositis"[Title/Abstract] \\
\hline \#34 & "radiation pneumonitis"[Title/Abstract] \\
\hline \#35 & "radiocystitis"[Title/Abstract] \\
\hline \#36 & "radiation proctitis"[Title/Abstract] \\
\hline \#37 & "radiation enteritis"[Title/Abstract] \\
\hline \#38 & "radiation esophagitis"[Title/Abstract] \\
\hline \#39 & "radiation encephalopathy"[Title/Abstract] \\
\hline$\# 40$ & "radiation ulcer" [Title/Abstract] \\
\hline$\# 41$ & $\begin{array}{l}\text { \#23 OR \#24 OR \#25 OR \#26 OR \#27 OR \#28 OR \#29 OR \#30 OR \#31 OR \#32 OR \#33 } \\
\text { OR \#34 OR \#35 OR \#36 OR \#37 OR \#38 OR \#39 OR \#40 }\end{array}$ \\
\hline \#42 & radiotherapy[MeSH Terms] \\
\hline$\# 43$ & "radiotherapy, computer-assisted"[MeSH Terms] \\
\hline \#44 & "radiotherapy, conformal"[MeSH Terms] \\
\hline$\# 45$ & chemoradiotherapy[MeSH Terms] \\
\hline \#46 & "cranial irradiation"[MeSH Terms] \\
\hline \#47 & "heavy ion radiotherapy"[MeSH Terms] \\
\hline \#48 & "radiotherapy dosage"[MeSH Terms] \\
\hline \#49 & "dose fractionation, radiation"[MeSH Terms] \\
\hline \#50 & "radiotherapy, image-guided"[MeSH Terms] \\
\hline$\# 51$ & "radiotherapy, high-energy"[MeSH Terms] \\
\hline$\# 52$ & "neutron capture therapy"[MeSH Terms] \\
\hline \#53 & radiodermatitis[MeSH Terms] \\
\hline$\# 54$ & $\begin{array}{l}\text { \#42 OR \#43 OR \#44 OR \#45 OR \#46 OR \#47 OR \#48 OR \#49 OR \#50 OR \#51 OR \#52 } \\
\text { OR \#53 }\end{array}$ \\
\hline \#55 & \#41 AND \#54 \\
\hline
\end{tabular}




\section{Appendix 2: Draft Eligibility Criteria}

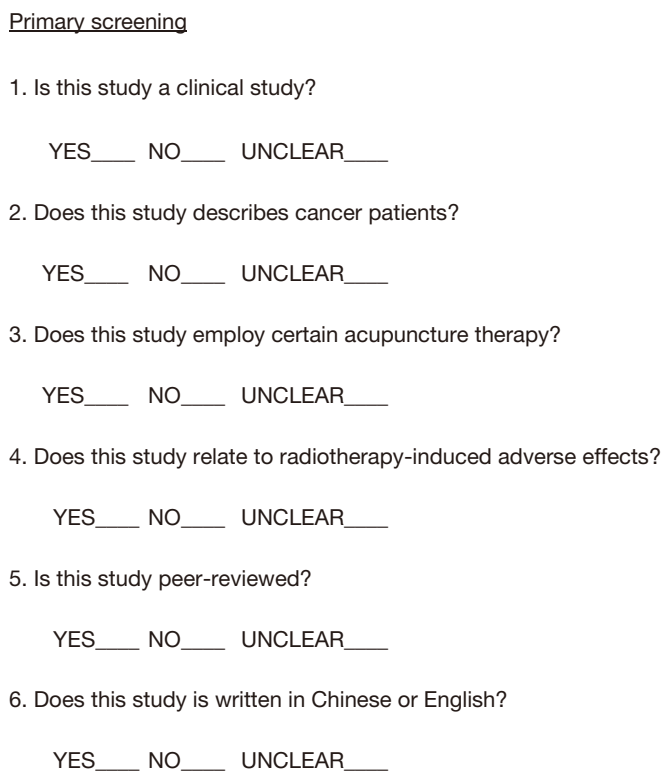

If you answer NO to any of these questions, the citation/study will be excluded. All other citations will be included.

Secondary Screening

1. Is this study a RCT?

YES NO_ UNCLEAR

2. Does patients in this study undergo single radiation therapy, not accompanied by chemotherapy or other interventions possibly causing adverse effects?

YES NO__ UNCLEAR

3. Is acupuncture therapy used as prophylaxis or treatment in this study?

YES NO UNCLEAR

4. Does this study use acupuncture therapy or combined therapies as intervention? YES NO UNCLEAR

5. Does this study record outcomes as required, such as the response rate of RIAE? YES $\mathrm{NO}$ UNCLEAR

6. Does sample size of each group in this study $>10$ ?

YES NO UNCLEAR

If you answer NO to any of these questions, the citation/study will be excluded. All other fulltext articles will be included. 